\title{
Penalized Maximum Likelihood Estimation for univariate normal mixture distributions
}

\author{
A. Ridolf* and J. Idier* \\ ${ }^{*}$ Laboratoire des Signaux et Systèmes (L2S), \\ 3 rue Joliot-Curie - Plateau de Moulon, 91192 Gif sur Yvette Cedex, France
}

\begin{abstract}
Due to singularities of the likelihood function, the maximum likelihood approach for the estimation of the parameters of normal mixture models is an acknowledged ill posed optimization problem. Ill posedness is solved by penalizing the likelihood function. In the Bayesian framework, it amounts to incorporating an inverted gamma prior in the likelihood function. A penalized version of the EM algorithm is derived, which is still explicit and which intrinsically assures that the estimates are not singular. Numerical evidence of the latter property is put forward with a test.
\end{abstract}

\section{INTRODUCTION}

Mixture models are a well fitted tool for clustering the observations together into groups for discrimination or classification : the mixture proportions then represent the relative frequency of occurrence of each group in the population. Mixture models also provide a convenient and flexible class of models for estimating or approximating distributions.

In particular, independent identically distributed (i.i.d.) mixture models well fit several problems in signal and image processing, covering a wide range of applications. In [1] a Bernoulli-Gaussian mixture model is adopted in a deconvolution problem, while [2] highlights the important role of mixture models in the field of cluster analysis. An example of the application of mixtures in biological (plant morphology measures) and physiological (EEG signals) data modeling is presented in [3]. Markovian mixture models are also commonly used, as in [4] where an application to medical image segmentation is considered.

The present contribution summarizes two of our previous works [5, 6], which focus on i.i.d. mixtures of univariate normal densities, with a known number of components. Parameters are estimated with a penalized maximum likelihood approach, by mean of the EM algorithm [7].

\section{MIXTURE MODEL}

We consider a sample $\boldsymbol{x}=\left\{x_{1}, \ldots, x_{T}\right\}$ of an i.i.d. mixture of $N$ univariate normal densities, with $N$ known

$$
f(x ; \boldsymbol{\theta})=\sum_{i=1}^{N} a_{i} f\left(x ; \mu_{i}, \sigma_{i}^{2}\right)
$$


where $f\left(x ; \mu_{i}, \sigma_{i}^{2}\right)=\left(2 \pi \sigma_{i}^{2}\right)^{-\frac{1}{2}} \exp \left\{-\left(x-\mu_{i}\right)^{2} / 2 \sigma_{i}^{2}\right\} . \boldsymbol{\theta}=\left\{\theta_{1}, \ldots, \theta_{N}\right\}$ are the mixture parameters, belonging to the parameter space

$$
\begin{aligned}
\Theta=\left\{\theta_{i}=\left\{a_{i}, \mu_{i}, \sigma_{i}^{2}\right\} \mid a_{i} \in \mathbb{R}_{+}, \sum_{i=1}^{N} a_{i}\right. & =1 ; \\
\sigma_{i}^{2} & \left.\in \mathbb{R}_{+} /\{0\} ; \mu_{i} \in \mathbb{R} \text { for } i=1, \ldots, N\right\}
\end{aligned}
$$

Given $\boldsymbol{x}$, the maximum likelihood estimate of the mixture parameters is defined as:

$$
\widehat{\boldsymbol{\theta}}_{T} \mid f\left(\boldsymbol{x} ; \widehat{\boldsymbol{\theta}}_{T}\right)=\sup _{\boldsymbol{\theta} \in \Theta} f(\boldsymbol{x} ; \boldsymbol{\theta})
$$

where

$$
f(\boldsymbol{x} ; \boldsymbol{\theta})=\prod_{k=1}^{T} f\left(x_{k} ; \boldsymbol{\theta}\right)=\prod_{k=1}^{T} \sum_{i=1}^{N} a_{i} f\left(x_{k} ; \mu_{i}, \sigma_{i}^{2}\right)
$$

is the likelihood function.

\section{LIKELIHOOD FUNCTION DEGENERACY}

Likelihood function degeneracy toward infinity is a well known problem for mixtures of Gaussian distributions, first put forward with a simple example in [8] (see also [9]). Such an example considered a two class mixture model with a corresponding likelihood function given by

$$
f(\boldsymbol{x} ; \boldsymbol{\theta})=\prod_{k=1}^{T}\left(\frac{a_{1}}{(2 \pi)^{\frac{1}{2}} \sigma_{1}} \exp \left\{-\frac{\left(x_{k}-\mu_{1}\right)^{2}}{2 \sigma_{1}^{2}}\right\}+\frac{a_{2}}{(2 \pi)^{\frac{1}{2}} \sigma_{2}} \exp \left\{-\frac{\left(x_{k}-\mu_{2}\right)^{2}}{2 \sigma_{2}^{2}}\right\}\right)
$$

Intuitively, the degeneracy is due to the fact that in the sum of Gaussian densities the variance parameter appears in the denominator. Indeed, couples such as $\left(\sigma_{i}^{2}=0, \mu_{i}=x_{k}\right)$ yield singularities, in the sense that $f$ tends to infinity as $\boldsymbol{\theta}$ approaches one of the corresponding points, located at the boundary of $\Theta$, as rigorously stated by the following property.

Property 1 Let us consider the likelihood function (3) for a given sample $\boldsymbol{x}=\left\{x_{1}, \ldots, x_{T}\right\}$, then

$$
\forall \boldsymbol{x} \in \mathbb{R}^{T}, \exists \boldsymbol{\theta}_{0} \in \bar{\Theta} \mid \lim _{\boldsymbol{\theta} \rightarrow \boldsymbol{\theta}_{0}} f(\boldsymbol{x} ; \boldsymbol{\theta})=+\infty
$$

where $\Theta$ is the parameter space (1), $\bar{\Theta}$ is the closure of the parameter space, $\boldsymbol{\theta}^{0}=$ $\left\{\boldsymbol{a}, \boldsymbol{\mu}, \boldsymbol{\sigma}^{2} \mid \exists i \in\{1, \ldots, N\}\right.$ and $\left.k \in\{1, \ldots, T\}, \mu_{i}=x_{k}, \sigma_{i}^{2}=0\right\} \in \bar{\Theta}$ is a point in the closure of the parameter space, and $\boldsymbol{\theta}=\left\{\boldsymbol{a}, \boldsymbol{\mu}, \boldsymbol{\sigma}^{2}\right\} \in \Theta$ is a point in the parameter space. 
Consequently, the maximum likelihood estimator (2) cannot be defined. In practice, unboundedness of $f(\boldsymbol{x} ; \boldsymbol{\theta})$ is a cause of failure of commonly used optimization algorithms, for instance of EM [9] and gradient types.

Hathaway [10] proposes to restrain the parameter space in order to exclude the points of degeneracy. The constrained maximization of the likelihood function is then numerically performed by a constrained EM algorithm [11]. However, such a constrained version of EM is complex to derive and to implement, with a resulting high computational cost.

Hence, we remain within the framework of the problem formulated on the whole parameter space (1). Moreover, we will specifically refer to the EM algorithm, which iteratively compute the maximum likelihood estimates by mean of the following reestimation formulas

$$
\begin{aligned}
a_{i}^{j+1} & =M_{i}\left(\boldsymbol{\theta}^{j}\right) / T \\
\mu_{i}^{j+1} & =M_{i}^{-1}\left(\boldsymbol{\theta}^{j}\right) \sum_{k=1}^{T} x_{k} \frac{a_{i}^{j} f\left(x_{k} ; \mu_{i}^{j}, \sigma_{i}^{2 j}\right)}{f\left(x_{k} ; \boldsymbol{\theta}^{j}\right)} \\
{\sigma_{i}^{2 j+1}}^{2 j} & =M_{i}^{-1}\left(\boldsymbol{\theta}^{j}\right) \sum_{k=1}^{T}\left(x_{k}-\mu_{i}^{j}\right)^{2} \frac{a_{i}^{j} f\left(x_{k} ; \mu_{i}^{j}, \sigma_{i}^{2 j}\right)}{f\left(x_{k} ; \boldsymbol{\theta}^{j}\right)}
\end{aligned}
$$

where $M_{i}\left(\boldsymbol{\theta}^{j}\right)=\sum_{k=1}^{T} a_{i}^{j} f\left(x_{k} ; \mu_{i}^{j}, \sigma_{i}^{2 j}\right) / f\left(x_{k} ; \boldsymbol{\theta}^{j}\right)$ and $j$ indicates the iteration.

\section{BAYESIAN SOLUTION TO DEGENERACY: PENALIZED LIKELIHOOD FUNCTION}

A Bayesian solution is proposed to solve the degeneracy of the likelihood function in the origin of any of the variance parameters. The latter are considered as i.i.d. random variables, leading to a penalized likelihood function

$$
f_{\mathrm{P}}(\boldsymbol{x}, \boldsymbol{\theta})=f\left(\boldsymbol{x}, \boldsymbol{\sigma}^{2} ; \boldsymbol{a}, \boldsymbol{\mu}\right)=f\left(\boldsymbol{x} \mid \boldsymbol{\sigma}^{2} ; \boldsymbol{a}, \boldsymbol{\mu}\right) \prod_{i=1}^{N} g\left(\sigma_{i}^{2}\right)
$$

where $g$ is the common prior probability density of variance parameters.

Our goal is to adjust $g$ so that the penalized likelihood is a bounded function that can be locally maximized by mean of an EM algorithm (which can be referred to as a "penalized" EM algorithm). In other words, $g$ must satisfy the requirements of

1. being a proper probability density function,

2. tending appropriately to zero to compensate for the likelihood singularities,

3. and allowing to maintain explicit re-estimation formulas for the resulting penalized EM algorithm. 
The inverted gamma distribution

$$
g\left(\sigma^{2}\right)=\frac{\alpha^{\beta-1}}{\Gamma(\beta-1)} \frac{1}{\sigma^{2^{\beta}}} \exp \left\{-\frac{\alpha}{\sigma^{2}}\right\} 1_{[0,+\infty)}
$$

which is known as the conjugate prior for the variance of a scalar Gaussian density [12], is proved to satisfy the three conditions.

As regard Point 1, the inverted gamma is assured to be proper by constraining the choice of its parameters: $\alpha>0$ and $\beta>1$, as discussed in [12].

As regard Point 2, the following property states the boundedness of $f_{\mathrm{P}}$ on $\Theta$ (whereas, from Property $1, f$ is an unbounded function under the same conditions), and it assures that the points of singularity do not maximize $f_{\mathrm{P}}$.

Property 2 The penalized likelihood is bounded above over the parameters space. Hence, the penalized likelihood function does not degenerate in any point of the closure of parameters space $\bar{\Theta}$. Moreover it tends to zero as $\sigma_{i}^{2} \rightarrow 0$, for any $i$. Hence, no $\sigma_{i}^{2}=0, i \in\{1 \ldots N\}$ maximizes the penalized likelihood function.

Proof For the sake of simplicity the proof refers to a two class mixture model, without loss of generality.

Akin to the likelihood function, the penalized version (8) may degenerate only in the origin of any of the parameters $\sigma^{2}$. Let us note $K=(2 \pi)^{-T / 2} \alpha^{2(\beta-1)} / \Gamma^{2}(\beta-1)^{2}$, and let us consider the likelihood function (4) penalized by a proper inverted gamma distribution (8)

$$
\begin{aligned}
& f_{\mathrm{P}}(\boldsymbol{x}, \boldsymbol{\theta})=K \frac{1}{\sigma_{1}^{2 \beta}} \exp \left\{-\frac{\alpha}{\sigma_{1}^{2}}\right\} \frac{1}{\sigma_{2}^{2 \beta}} \exp \left\{-\frac{\alpha}{\sigma_{2}^{2}}\right\} \\
& \prod_{k=1}^{T}\left(\frac{a_{1}}{\sigma_{1}} \exp \left\{-\frac{\left(x_{k}-\mu_{1}\right)^{2}}{2 \sigma_{1}^{2}}\right\}+\frac{a_{2}}{\sigma_{2}} \exp \left\{-\frac{\left(x_{k}-\mu_{2}\right)^{2}}{2 \sigma_{2}^{2}}\right\}\right)
\end{aligned}
$$

On every compact domain strictly contained in the parameter space, $f_{\mathrm{P}}$ is bounded. This is a straightforward consequence of the fact that $f_{\mathrm{P}}$ is the product of two functions which are bounded on such domains (the product of sum of Gaussian distributions and the product of inverted gamma distributions). Hence, it is sufficient to prove that $f_{\mathrm{P}}$ remains bounded on the boundaries of $\Theta$, and more precisely that it remains bounded in the points of singularity. 
From the inequality $\exp \left\{-(x-\mu)^{2} / 2 \sigma^{2}\right\} \leq 1$ the likelihood function can be bounded above by

$$
\begin{aligned}
<K \frac{1}{\sigma_{1}^{2 \beta}} \exp \left\{-\frac{\alpha}{\sigma_{1}^{2}}\right\} \frac{1}{\sigma_{2}^{2 \beta}} \exp \left\{-\frac{\alpha}{\sigma_{2}^{2}}\right\} \prod_{k=1}^{T}\left(\frac{1}{\sigma_{1}}+\frac{1}{\sigma_{2}}\right) \\
=K \prod_{k=1}^{T}\left(\frac{1}{\sigma_{1}^{2\left(\frac{\beta}{T}+\frac{1}{2}\right)}} \exp \left\{-\frac{\alpha}{T \sigma_{1}^{2}}\right\} \frac{1}{\sigma_{2}^{2 \frac{\beta}{T}}} \exp \left\{-\frac{\alpha}{T \sigma_{2}^{2}}\right\}\right. \\
\left.+\frac{1}{\sigma_{1}^{2 \frac{\beta}{T}}} \exp \left\{-\frac{\alpha}{T \sigma_{1}^{2}}\right\} \frac{1}{\sigma_{2}^{2\left(\frac{\beta}{T}+\frac{1}{2}\right)}} \exp \left\{-\frac{\alpha}{T \sigma_{2}^{2}}\right\}\right)
\end{aligned}
$$

By considering that

$$
\lim _{\sigma^{2} \rightarrow 0} \frac{1}{\sigma^{2\left(\frac{\beta}{T}+\frac{1}{2}\right)}} \exp \left\{-\frac{\alpha}{T \sigma^{2}}\right\}=0
$$

and that

$$
\lim _{\sigma^{2} \rightarrow 0} \frac{1}{\sigma^{2 \frac{\beta}{T}}} \exp \left\{-\frac{\alpha}{T \sigma^{2}}\right\}=0
$$

it is straightforward to see that the penalized likelihood function tends to zero as $\sigma^{2} \rightarrow 0$. Therefore, it is bounded in the point of singularity and its boundedness on the whole parameter space follows.

Hence, the existence of the penalized maximum likelihood estimator is assured, and such an estimator falls within the parameters space $\Theta$ (the boundaries are excluded by the null value of the likelihood).

Moreover, the penalized likelihood estimator has recently been proved to be consistent [6].

\section{PENALIZED EM ALGORITHM}

As regard Point 3, explicitness directly follows from the appropriate choice of $g\left(\sigma^{2}\right)$ as an inverted gamma distribution. Indeed, the EM algorithm is based on the iterative maximization, with respect to $\boldsymbol{\theta}$, of a criterion $Q$, which, at iteration $j+1$, is given by

$$
Q\left(\boldsymbol{\theta}, \boldsymbol{\theta}^{j} ; \boldsymbol{x}\right) \int f\left(\boldsymbol{c} \mid \boldsymbol{x} ; \boldsymbol{\theta}^{j}\right) \ln f(\boldsymbol{x}, \boldsymbol{c} ; \boldsymbol{\theta}) d \boldsymbol{c}=\mathrm{E}\left[\ln f(\boldsymbol{x}, \boldsymbol{C} ; \boldsymbol{\theta}) \mid \boldsymbol{x} ; \boldsymbol{\theta}^{j}\right]
$$

where $\boldsymbol{c}=\left\{c_{k} \in\{1, \ldots, N\}, k=1, \ldots, T\right\}$ are the classes of the elements of the sample $\boldsymbol{x}$.

Considered as a function of $\sigma^{2}, f(\boldsymbol{x}, \boldsymbol{c} ; \boldsymbol{\theta})=f\left(\boldsymbol{x} \mid \boldsymbol{c} ; \boldsymbol{\sigma}^{2}, \boldsymbol{\mu}\right) \mathrm{P}(\boldsymbol{c} ; \boldsymbol{a})$, with $\mathrm{P}(\boldsymbol{c} ; \boldsymbol{a})=\prod_{k=1}^{T} a_{c_{k}}$, is proportional to the likelihood function of the complete data

$$
f(\boldsymbol{x} \mid \boldsymbol{c} ; \boldsymbol{\theta})=\prod_{k=1}^{T} f\left(x_{k} ; \mu_{c_{k}}, \sigma_{c_{k}}^{2}\right)
$$


which is a product of Gaussian densities. With respect to $\sigma^{2}$, the likelihood function of the complete data (12) also reads

$$
\begin{aligned}
f(\boldsymbol{x} \mid \boldsymbol{c} ; \boldsymbol{\theta}) & =\prod_{i=1}^{N} G\left(\sigma_{i}^{2} ; h_{i}, l_{i}, \gamma_{i}\right) \\
G\left(\sigma^{2} ; h, l, \gamma\right) & =\frac{h}{\sigma^{\gamma}} \exp \left\{-\frac{l^{2}}{\sigma^{2}}\right\}
\end{aligned}
$$

where $T_{i}$ denotes the number of data sampled from the class $i$,

$$
h_{i}=(2 \pi)^{-T_{i} / 2} ; \quad l_{i}^{2}=\sum_{k \mid c_{k}=i}\left(x_{k}-\mu_{i}\right)^{2} ; \quad \gamma_{i}=T_{i}
$$

and $G\left(\sigma_{i}^{2} ; h_{i}, l_{i}, \gamma_{i}\right)=1$ if $T_{i}=0, i=1, \ldots, N$.

By applying the penalization, the term $f(\boldsymbol{x}, \boldsymbol{c} ; \boldsymbol{\theta})$ of (11) changes to

$$
f(\boldsymbol{x}, \boldsymbol{c} ; \boldsymbol{\theta}) g\left(\boldsymbol{\sigma}^{2}\right)=f\left(\boldsymbol{x} \mid \boldsymbol{c}, \boldsymbol{\sigma}^{2} ; \boldsymbol{\mu}\right) g\left(\boldsymbol{\sigma}^{2}\right) \mathrm{P}(\boldsymbol{c} ; \boldsymbol{a})
$$

where, as a function of $\sigma^{2}$,

$$
f\left(\boldsymbol{x} \mid \boldsymbol{c}, \boldsymbol{\sigma}^{2} ; \boldsymbol{\mu}\right) g\left(\boldsymbol{\sigma}^{2}\right)=\prod_{i=1}^{N} G\left(\sigma_{i}^{2} ; h_{i}^{\prime}, l_{i}^{\prime}, \gamma_{i}^{\prime}\right)
$$

with

$$
h_{i}^{\prime}=\alpha^{\beta-1} \Gamma(\beta-1)^{-1}(2 \pi)^{-T_{i} / 2} ; \quad l_{i}^{\prime 2}=\alpha+\sum_{k \mid c_{k}=i}\left(x_{k}-\mu_{i}\right)^{2} ; \quad \gamma_{i}^{\prime}=2 \beta+T_{i}
$$

Therefore, penalization by an inverted gamma distribution has introduced no "structural" changes in criterion $Q$ of the EM algorithm and explicitness is maintained.

A more thorough analysis reveals that the re-estimation equations remain explicit because $g$ is chosen as the conjugate prior of the likelihood of the complete data ([13], [12, page 99]).

The re-estimation equations of the penalized EM algorithm are not only explicit, but they also correspond to a very slight alteration of the standard ones. Indeed, equations (5) and (6) remain unchanged, while equation (7) becomes

$$
{\sigma_{i}^{2 j+1}}^{j}=\frac{2 \alpha+\sum_{k=1}^{T}\left(x_{k}-\mu_{i}^{j}\right)^{2} a_{i}^{j} f\left(x_{k} ; \mu_{i}^{j}, \sigma_{i}^{2 j}\right) / f\left(x_{k} ; \boldsymbol{\theta}^{j}\right)}{2 \beta+M_{i}\left(\boldsymbol{\theta}^{j}\right)}
$$

Therefore, penalization of the EM does not increase the computational burden: this is an extremely important aspect in the case of large samples or image processing. 
Moreover, from equation (13) it is straightforward to see that every maximizer (either global or local) of the penalized likelihood function yields strictly positive variance estimates. Indeed we have

$$
\forall j \quad \sigma_{i}^{2 j} \geq \sigma_{\min }^{2}(T)=\frac{2 \alpha}{2 \beta+T}>0
$$

where $\sigma_{\min }^{2}(T)$ tends to 0 as $T$ tends to infinity.

\section{NUMERICAL RESULTS}

We have tested the penalized and non penalized EM algorithm on a 2 class mixture model, defined in (4).

Eight-hundred samples of length fifty have been randomly generated from two Gaussian distribution, having parameters $\boldsymbol{a}=\left[\begin{array}{ll}0.5 & 0.5\end{array}\right], \boldsymbol{\mu}=\left[\begin{array}{ll}0 & 2.5\end{array}\right], \boldsymbol{\sigma}^{2}=\left[\begin{array}{ll}1 & 2\end{array}\right]$.

For each sample, the starting point of the EM iterations was chosen automatically. Such a choice is based on partitioning the empirical histogram of the data, as proposed in [14]. As in [11], the EM algorithm was considered to have converged whenever the maximum of the relative stepsize

$$
\left|a_{i}{ }^{j+1}-a_{i}{ }^{j}\right| / a_{i}{ }^{j},\left|\mu_{i}{ }^{j+1}-\mu_{i}{ }^{j}\right| / \mu_{i}{ }^{j},\left|{\sigma_{i}^{2 j+1}}^{j+1}-\sigma_{i}^{2^{j}}\right| / \sigma_{i}^{2^{j}}
$$

for $i=1 \ldots N$, became less or equal than $10^{-5}$.

Figure 1 depicts the histograms for the values of the non-penalized estimates of $\sigma_{1}^{2}$ and $\sigma_{2}^{2}$, and the histograms for the values of the penalized ones.

By comparing the histograms, the efficiency of penalization becomes evident. Without penalization, the distribution of the estimates spreads toward the singularity $\left(\sigma^{2}=0\right.$, hence $\log \sigma^{2}=-\infty$ ), and for 13 times the EM algorithm converges to the singularity itself. On the other hand, coherently with the theoretical results of Property 2, the estimates computed by the penalized EM algorithm are concentrated around the true value and none of them is a singularity.

By increasing the length of the samples the number of convergence of the standard EM algorithm to singularities is reduced (probably as a consequence of a restriction of the attracting domain of the degeneracy point), but it is still greater than zero. Table 1 summarizes the results for samples of length fifty and one-hundred of the non-penalized (a) and penalized (b) EM algorithm.

TABLE 1. Non penalized ( $a$ ) and penalized $(b)$ EM algorithm results for samples of length fi fty and one-hundred.

(a)

\begin{tabular}{||c||c||}
\hline 800 samples of length: & convergence to singularities: \\
\hline 50 & 13 \\
\hline 100 & 1 \\
\hline
\end{tabular}

(b)

\begin{tabular}{||c||c||}
\hline 800 samples of length: & min value of $\sigma^{2}:$ \\
\hline 50 & 0.3951 \\
\hline 100 & 0.4247 \\
\hline
\end{tabular}



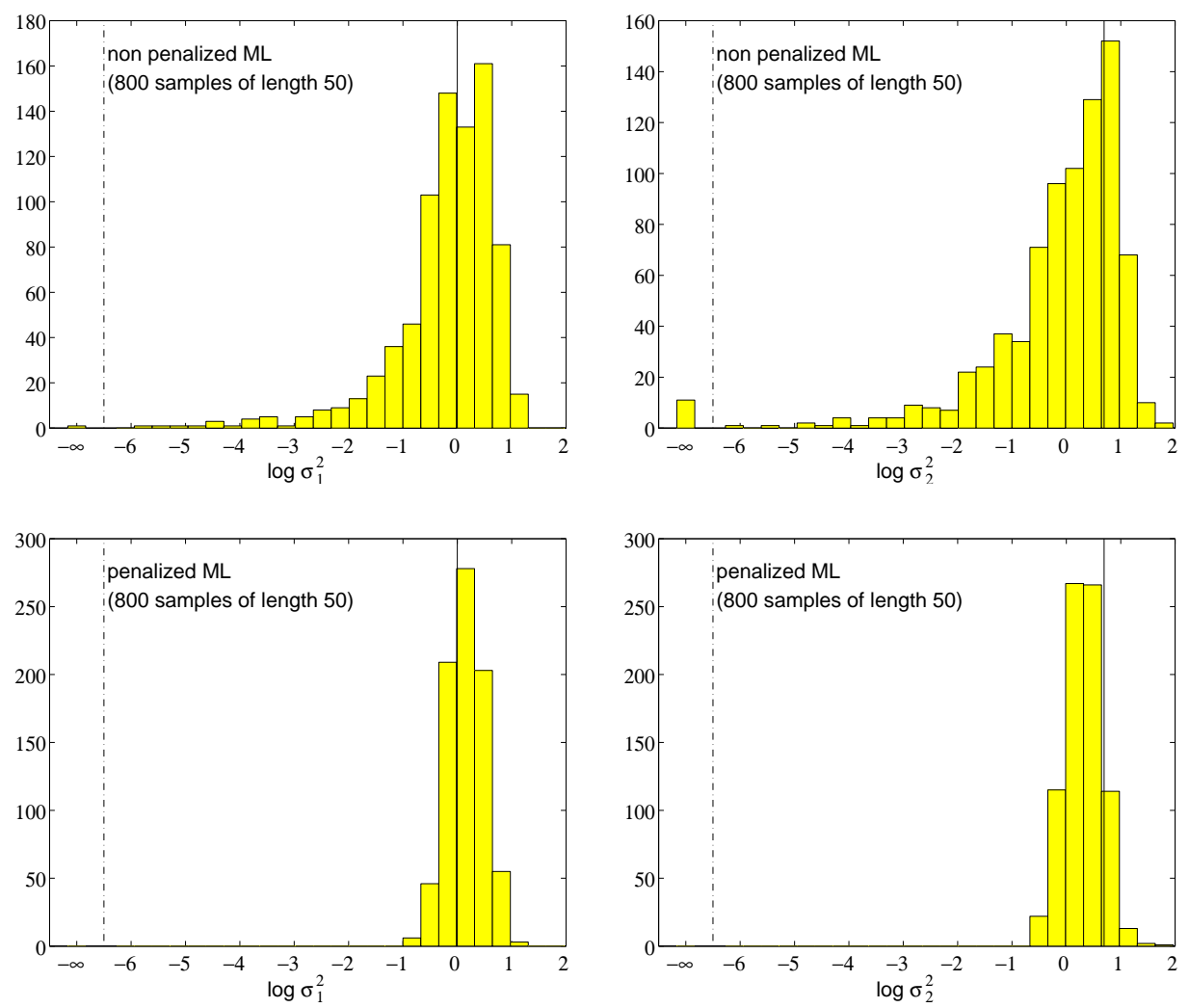

FIGURE 1. Histograms of EM $\sigma_{1}^{2}$ and $\sigma_{2}^{2}$ estimates, where the solid line indicates the true value while the dashed line indicates a rupture toward infi nity of the $x$ axis. The top two histograms and the bottom ones refer to the values of the non-penalized and penalized estimates, respectively. Penalization evidently avoids spreading toward the singularity $\left(\sigma^{2}=0\right.$, hence $\left.\log \sigma^{2}=-\infty\right)$ of the $\sigma^{2}$ estimates.

\section{CONCLUDING REMARKS}

Penalization of the likelihood has revealed itself to be an efficient and simple solution to likelihood degeneracy.

Theoretical properties ensure the existence of the maximum likelihood estimator as well as its belonging to the parameter space.

The choice of the conjugate prior of the likelihood of the complete data as penalization term conducted to explicit EM algorithm re-estimation formulas. While the role of conjugate priors is acknowledged in Bayesian sampling schemes, including mixture problems [13], putting forward the link between conjugate priors and explicit penalized EM schemes is an original contribution, as far as we know.

Numerical examples evidence the existence of singularities and the efficiency of the penalized solution.

Concerning the asymptotic behavior of the penalized maximum likelihood estimator, we know from [15] that penalization does not alter asymptotic properties such as consistency. Hence, local consistency of the penalized estimator is a direct consequence of local consistency of the non penalized one (see [15]). On the other hand, global con- 
sistency cannot be similarly deduced, since the non penalized maximum likelihood estimator is globally not even defined and classical theorems, as [16] and [8], cannot be applied. Although not trivial, proof of global consistency has recently been achieved [6].

To our best knowledge, Hathaway's EM re-estimation formulas [11] are the only preexisting non-degenerate alternative to our penalized version. It is based on constrained maximization of the likelihood, within an appropriately chosen subset of $\Theta$. However, Hathaway's version is substantially more complex to derive and to implement, and the resulting numerical cost is higher.

\section{REFERENCES}

1. F. Champagnat, Y. Goussard, and J. Idier, 'Unsupervised deconvolution of sparse spike trains using stochastic approximation,"IEEE Trans. Signal Processing, 44, pp. 2988-2998, December 1996.

2. G. J. McLachlan and K. E. Basford, Mixture Models, inference and applications to clustering, vol. 84 of statistics, Dekker, 1987.

3. S. J. Roberts, D. Husmeier, I. Rezek, and W. Penny, 'Bayesian approaches to Gaussian mixture modeling," IEEE Trans. Pattern Anal. Mach. Intell., 20, pp. 887-906, November 1998.

4. A. Ridolfi, Maximum Likelihood Estimation of Hidden Markov Model Parameters, with Application to Medical Image Segmentation. Tesi di Laurea, Politecnico di Milano, Facoltà di Ingegneria, Milano, Italia, 1997.

5. A. Ridolfi and J. Idier, 'Penalized maximum likelihood estimation for univariate normal mixture distributions," in Actes du $17^{\text {e }}$ colloque GRETSI, (Vannes, France), pp. 259-262, September 1999.

6. G. Ciuperca, A. Ridolfi , and J. Idier, 'Penalized maximum likelihood estimator for normal mixtures," Prepublication 2000-70, Laboratoire de Probabilités, Statistiques et Modélisation, Orsay, France, and L2S, Gif-sur-Yvette, France, 2000.

7. A. P. Dempster, N. M. Laird, and D. B. Rubin, 'Maximum likelihood from incomplete data via the EM algorithm," J. R. Statist. Soc. B, 39, pp. 1-38, 1977.

8. J. Kiefer and J. Wolfowitz, "Consistency of the maximum likelihood estimator in the presence of infi nitely many incidental parameters,” Ann. Math. Statist., 27, pp. 887-906, 1956.

9. R. A. Redner and H. F. Walker, "Mixture densities, maximum likelihood and the EM algorithm," SIAM Rev., 26, pp. 195-239, April 1984.

10. R. J. Hathaway, "A constrained formulation of maximum-likelihood estimation for normal mixture distributions," The Annals of Statistics, 13, pp. 795-800, 1985.

11. R. J. Hathaway, "A constrained EM algorithm for univariate normal mixtures," J. Statist. Comput. Simul., 23, pp. 211-230, 1986.

12. C. Robert, L'analyse statistique Bayésienne, Economica, 1992.

13. J. Diebolt and C. P. Robert, 'Estimation of fi nite mixture distributions through Bayesian sampling," J. R. Statist. Soc. B, 56, (2), pp. 363-375, 1994.

14. P. A. Devijver and M. Dekessel, 'Champs aléatoires de Pickard et modélisation d'images digitales," Traitement du Signal, 5, (5), pp. 131-150, 1988.

15. R. A. Redner, 'Maximum likelihood estimation for mixture models," technical memorandum, NASA, October 1980.

16. A. Wald, "Note on the consistency of the maximum likelihood estimate," Ann. Math. Stat., 20, pp. 595-601, 1949. 